Linköping Studies in Science and Technology.

Dissertations, No. 1824

\title{
High-order finite difference approximations for hyperbolic problems: multiple penalties and non-reflecting boundary conditions
}

Hannes Frenander

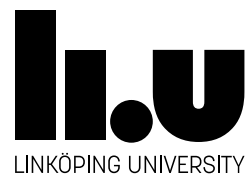

Department of Mathematics, Division of Computational Mathematics Linköping University, SE-581 83 Linköping, Sweden

Linköping 2017 
Linköping Studies in Science and Technology. Dissertations, No. 1824

High-order finite difference approximations for hyperbolic problems: multiple penalties and non-reflecting boundary conditions

Copyright @ C Hannes Frenander, 2017

Division of Computational Mathematics

Department of Mathematics

Linköping University

SE-581 83, Linköping, Sweden

hannes.frenander@liu.se

www.liu.se/mai/ms

Typeset by the author in $\mathrm{H}_{\mathrm{T}} \mathrm{T}$ Xe documentation system.

ISSN 0345-7524

ISBN 978-91-7685-595-9

Printed by LiU-Tryck, Linköping, Sweden 2016 


\begin{abstract}
In this thesis, we use finite difference operators with the Summation-By-Parts property (SBP) and a weak boundary treatment, known as Simultaneous Approximation Terms (SAT), to construct high-order accurate numerical schemes. The SBP property and the SAT's makes the schemes provably stable. The numerical procedure is general, and can be applied to most problems, but we focus on hyperbolic problems such as the shallow water, Euler and wave equations.

For a well-posed problem and a stable numerical scheme, data must be available at the boundaries of the domain. However, there are many scenarios where additional information is available inside the computational domain. In terms of well-posedness and stability, the additional information is redundant, but it can still be used to improve the performance of the numerical scheme. As a first contribution, we introduce a procedure for implementing additional data using SAT's; we call the procedure the Multiple Penalty Technique (MPT).
\end{abstract}

A stable and accurate scheme augmented with the MPT remains stable and accurate. Moreover, the MPT introduces free parameters that can be used to increase the accuracy, construct absorbing boundary layers, increase the rate of convergence and control the error growth in time.

To model infinite physical domains, one need transparent artificial boundary conditions, often referred to as Non-Reflecting Boundary Conditions (NRBC). In general, constructing and implementing such boundary conditions is a difficult task that often requires various approximations of the frequency and range of incident angles of the incoming waves. In the second contribution of this thesis, we show how to construct NRBC's by using SBP operators in time.

In the final contribution of this thesis, we investigate long time error bounds for the wave equation on second order form. Upper bounds for the spatial and temporal derivatives of the error can be obtained, but not for the actual error. The theoretical results indicate that the error grows linearly in time. However, the numerical experiments show that the error is in fact bounded, and consequently that the derived error bounds are probably suboptimal. 



\section{Sammanfattning på svenska}

Många fenomen som observeras i naturen beskrivs matematiskt av partiella differentialekvationer med lämpliga rand-och initialvillkor. Dessa fenomen påträffas inom flödesdynamik, kvantmekanik och elektromagnetism, för att nämna några exempel. I allmänhet kan dessa problem inte lösas analytiskt, och måste därför modelleras med hjälp av datorer. Den spatiella domänen delas då upp i ett ändligt antal diskreta punkter, där man söker en approximativ lösning.

I den här avhandlingen används finita differensoperatorer på s.k partiell summationsform för att konstruera noggranna numeriska metoder. Med hjälp av en svag implementation av randvillkoren blir metoden även stabil. Denna metodik är allmän och kan tillämpas på alla problem, men i denna avhandling fokuserar vi på våg- och flödesproblem.

Data måste vara tillgängligt på randen för att problemet ska vara välställt. Ytterligare information om lösningen kan emellertid vara tillgänglig inuti domänen. Denna ytterligare information är överflödig när det gäller att konstruera ett noggrant och stabilt numeriskt schema, men kan likväl användas för att förbättra prestandan på olika sätt. Som ett första bidrag introduceras en metod för att inkorporera tillgängligt data i domänen, så att metoden förblir stabil. Vi kallar denna metod för "multiple penalty technique" (MPT). Med denna teknik introduceras ett antal fria parametrar som kan användas för att göra metoden noggrannare, öka konvergenshastigheten, konstruera absorberande randskikt och kontrollera feltillväxten hos vissa problem.

I många tillämpningar måste oändliga domäner modelleras, och domänen måste begränsas med hjälp av s.k artificiella randvillkor. Dessa randvillkor bör vara transparenta, d.v.s all information som träffar randen ska transporteras ut ur domänen utan att ge upphov till reflektioner. Fullständigt transparenta randvillkor kallas för icke-reflekterande randvillkor. Det andra bidraget i denna avhandling går ut på att konstruera och implementera denna typ av randvillkor med hjälp av tidsoperatorer på partiell summationsform. Randvillkoren ger ett välställt problem och det numeriska schemat är stabilt.

Som ett sista bidrag i denna avhandling studeras felgränser för vågekvationen på andra ordningens form. Övre gränser för rums-och tidsderivatan för felet erhålls, men inte för själva felet. De teoretiska resultaten indikerar att felet växer linjärt med tiden, medan numeriska experiment indikerar att en övre felgräns existerar. 



\section{Acknowledgments}

First and foremost, I would like to thank my supervisor Prof. Jan Nordström for his excellent guidance and support, and for patiently guiding me through the challenges as a Ph.D student. His level of commitment has been far beyond expectations. Without his expertise and feedback, most of my work would not have been possible.

I would like to express my gratitude towards my colleagues at MAI for valuable discussions, advice and feedback, and for making my time as a Ph.D student enjoyable. Especially, I would like to thank my fellow Ph.D students for proofreading my articles and providing excellent feedback.

The support from administrative staff on MAI has been invaluable. Especially, I would like to thank Theresia, Madelaine and Monika for helping me with the, sometimes confusing, administrative issues during my time as a Ph.D student.

Finally, I would like to thank my family and friends for their love and encouragement! Especially, I want to express my love and gratitude towards Klara Kemmer, who have always been there for me.

This work was partially funded by the Swedish e-science Research Centre (SeRC). 



\section{List of Papers}

This thesis is based on the following papers, which will be referred to in the text by their roman numerals.

I. J. Nordström, Q. Abbas, B. Erickson and H. Frenander, A flexible boundary procedure for hyperbolic problems: multiple penalty terms applied in a domain, Communications in Computational Physics 16 (2014) 541-570.

II. H. Frenander and J. Nordström, A stable and accurate Davies-like relaxation procedure using multiple penalty terms for lateral boundary conditions, Dynamics of Atmospheres and Oceans 73 (2016) 34-46.

III. H. Frenander and J. Nordström, A stable and accurate data assimilation technique using multiple penalty terms in space and time, Submitted (2016).

IV. H. Frenander and J. Nordström, Constructing non-reflecting boundary conditions using summation-by-parts in time, Journal of Computational Physics 331 (2017) 38-48.

V. J. Nordström, and H. Frenander, Long time error bounds for the wave equation on second order form, Technical report, LiTH-MAT-R-2017/01SE (2017).

In Paper I, I developed parts of the theory, conducted some of the numerical experiments and wrote parts of the manuscript. I developed most of the theoretical results in Paper II-IV, performed all numerical experiments and wrote the manuscript with editorial support from Prof. Jan Nordström. The theoretical analysis of Paper $\mathrm{V}$ was developed jointly with the first author. I performed the numerical experiments and the manuscript was written by both the first and second author. 



\section{Contents}

Abstract i

Sammanfattning på svenska

Acknowledgments v v

List of Papers vii

1 Introduction 1

1.1 Outline of this thesis . . . . . . . . . . . . 3

2 The SBP-SAT technique 5

2.1 The continous problem . . . . . . . . . . . . 5

2.1.1 A model problem ............... 6

2.2 The discrete problem . . . . . . . . . . . . . . . . . 7

2.2.1 The one-dimensional SBP-SAT technique . . . . . . 8

2.2.2 The multi-dimensional SBP-SAT technique . . . . . . . 9

3 The multiple penalty technique 11

3.1 Increasing the rate of convergence . . . . . . . . . . . . . . . 12

3.2 Increasing the accuracy . . . . . . . . . . . . . . . . 13

3.3 Modifying the wave speed of the error . . . . . . . . . . . 15

3.4 Constructing absorbing boundary layers . . . . . . . . . . . . 15

3.5 The MPT in several space dimensions . . . . . . . . . . . . . 16

3.6 Controlling error growth . . . . . . . . . . . . . . . . . . . . . . 18

3.7 Conclusions and future outlook . . . . . . . . . . . . . 19

4 Non-reflecting boundary conditions $\quad 21$

4.1 NRBC's in one dimension . . . . . . . . . . . . . . . . . 21

4.2 NRBC's for systems of hyperbolic equations . . . . . . . . . . . . 22

4.2.1 NRBC's for one-dimensional hyperbolic problems . . . . . 25

4.3 The new way to construct NRBC's . . . . . . . . . . . . . . . 25

4.3.1 Numerical verification . . . . . . . . . . . . . . . 27

4.4 Conclusions and future outlook . . . . . . . . . . . . . 28 
5 Error bounds for the wave equation 31

5.1 Conclusions and future outlook . . . . . . . . . . . . . . . 32

6 Summary of papers $\quad 35$

$\begin{array}{ll}\text { References } & 38\end{array}$

\section{INCLUDED PAPERS}

I. A flexible boundary procedure for hyperbolic problems: multiple penalty terms applied in a domain . . . . . . . . . . . . . 41

II. A stable and accurate Davies-like relaxation procedure using multiple penalty terms for lateral boundary conditions . . . . . . . . 73

III. A stable and accurate data assimilation technique using multiple penalty terms in space and time . . . . . . . . . . . . 89

IV. Constructing non-reflecting boundary conditions using summationby-parts in time . . . . . . . . . . . . . . . . . . 109

V. Long time error bounds for the wave equation on second order form 123 


\section{Introduction}

A considerable amount of all phenomena in nature are described by Partial Differential Equations (PDE's) together with appropriate initial and boundary conditions. This includes the Maxwell equations in electrodynamics, the Dirac and Schrödinger equation in quantum mechanics and the Navier-Stokes equations in fluid dynamics, among many other examples. Needless to say, finding solutions to Initial Boundary Value Problems (IBVP's) (PDE's together with appropriate initial and boundary conditions) is very important in both science and engineering. Unfortunately, finding exact solutions to IBVP's is in general only possible under very simplified conditions; for more realistic scenarios, the problem must be approximated using numerical algorithms.

The first issue to consider when constructing a numerical scheme is that the IBVP's is formulated correctly. If inappropriate initial and boundary conditions are used, the numerical solution will be unreliable. More precisely, the boundary treatment must be done such that the IBVP is well-posed, i.e. such that there exist a unique solution that depends continuously on the applied data. Once a well-posed problem is available, the numerical scheme must be consistent, i.e. it must be an accurate approximation of the IBVP. The numerical scheme must also be stable, which means that the growth of the solution is limited by applied data. If the scheme is both stable and accurate, the numerical solution will converge to the continous one during mesh refinement.

Finite difference operators with the Summation-By-Parts (SBP) property have been used since the 1970's, and are used to construct high-order accurate and provable stable schemes $[13,21,26,19]$. As we will discuss later, the SBP operators are constructed to mimic integration-by-parts, which is the most important technique for obtaining a well-posed problem. Throughout this thesis, we will make use of the SBP operators when constructing numerical schemes. For implementing the boundary conditions, we use a weak formulation known as the Simultaneous Approximation Term (SAT) technique [4, 5].

To obtain a well-posed problem and a stable scheme, data must be available at the boundaries of the domain. However, additional data inside the computational domain may also be available. This is often the case when making weather predictions, where observations of, for example, temperature and air pressure from weather stations can be inserted into ongoing simulations. A popular technique for this so-called data assimilation is the 3D Variational (3D-Var) and 4D 
Variational (4D-Var) approach [6, 8]. In these methods, an appropriate initial condition based on the observations is constructed by finding a minima to a cost function. As a first contribution of this thesis, we show how to implement additional data inside the domain using SAT's such that the scheme remains provably stable. We refer to this technique as the Multiple Penalty Technique (MPT). As will be shown later, the MPT introduces free parameters that can be used to improve the performance of the numerical scheme. For example, the MPT can be used the create absorbing boundary layers, increase the rate of convergence, increase the accuracy of the scheme and control the error growth for certain problems.

Another scenario often encountered in science and engineering are infinite physical domains. For obvious reasons, one need to limit the domain in the numerical model by introducing Artificial Boundary Conditions (ABC's). To accurately mimic the physical problem, the ABC's must be as transparent as possible; that is, information that propagates towards the artificial boundary should ideally pass through without being reflected back into the domain. Completely transparent ABC's are referred to as Non-Reflecting Boundary Conditions (NRBC's). Unfortunately, constructing NRBC's for a general problem in more than one space dimension is not an easy task, and one often have to resort to approximations of various parameters of the incoming waves when constructing them. This subject is treated in the classical paper by Engquist and Majda [9] and in $[25,14,11,28,10]$. Another way to construct NRBC's is to add buffer zones, where the incoming waves are eliminated. A popular method the utilizes this technique is called Perfectly Matched Layers (PML) [3, 1, 15]. As a second contribution of this thesis, we show how to use the SBP-SAT technique in time $[20,23,24]$ to construct exact NRBC's. Our technique is performed in timedomain and it is based on the analysis performed in $[9,25,10]$. It is shown that the derived NRBC's result in a well-posed problem and that the corresponding numerical scheme is stable and accurate.

A well-posed problem and a stable numerical scheme may experience a growth of the error during long time simulations $[22,17]$. In the final contribution of this thesis, long time error bounds for the wave equation on second order form is investigated. The analysis yields a bound on the space and time derivatives of the error while the actual error grows linearly in time. However, numerical tests indicate that also the error is bounded, and that the theoretically predicted linear growth stems from non-sharp estimates. 


\subsection{Outline of this thesis}

In Chapter 2, we describe the method used in this thesis for constructing numerical schemes, namely the SBP-SAT technique. We show how boundary and initial conditions must be imposed to produce a well-posed problem, how the SBP operators are constructed and how the SBP-SAT technique leads to a highorder accurate and stable scheme. Next, in Chapter 3, we describe and discuss the MPT. The content of that chapter is based on Paper I, II and III, and the theoretical findings in these papers are summarized together with the most important numerical results. A new method for constructing and implementing NRBC's was introduced in Paper IV, and that paper is summarized in Chapter 4. First, the general theory of how to construct NRBC's is described. Secondly, the theoretical findings will be discussed, and some of the numerical results will be displayed. In Chapter 5, error bounds for the wave equation on second order form is investigated; the contents are based on the results of Paper V. Error bounds for long time simulations are derived and compared with the numerical results. Finally, in Chapter 6, we give a brief summary of the content of the papers included in this thesis. 



\section{The SBP-SAT technique}

The main advantage of the SBP-SAT technique is its stability properties. In fact, as long as the IBVP is well-posed, stability of the numerical scheme follows almost automatically when using the SBP-SAT technique. Moreover, high order accurate schemes are as easily obtained as low order ones. In this chapter, we will discuss how the SBP operators are constructed, and how to obtain stable and high-order accurate schemes.

\subsection{The continous problem}

Before moving on to the discretization of IBVP's and the SBP-SAT technique, we shortly discuss how the continous problem should be formulated. Consider a general linear IBVP on the form,

$$
\begin{aligned}
u_{t}(\bar{x}, t) & =H u(\bar{x}, t)+F(\bar{x}, t), \quad \bar{x} \in \Omega, t \geq 0 \\
L u(\bar{x}, t) & =g(t), \quad \bar{x} \in \delta \Omega, t \geq 0 \\
u(\bar{x}, 0) & =f(\bar{x}), \quad \bar{x} \in \Omega, t=0
\end{aligned}
$$

where $H$ is a differential operator, $L$ a boundary operator, $F, g, f$ given data and $\bar{x}$ the position vector. Throughout this thesis, we use subscripts to denote partial derivatives, i.e. $u_{t}=\partial u / \partial t$ in (2.1). The spatial domain under consideration is denoted $\Omega$ with boundary $\delta \Omega$.

The problem (2.1) is well-posed if there exist a unique solution that depends continuously on the applied data $F, g$ and $f$. Assuming that a solution $u$ to (2.1) exist, the problem is strongly well-posed if $u$ satisfies an estimate on the form,

$$
\|u\|_{1}^{2} \leq C e^{\alpha t}\left(\|f\|_{2}^{2}+\int_{0}^{t}\left(\|F\|_{3}^{2}+\|g\|_{4}^{2}\right) d \tau\right),
$$

where $C$ and $\alpha$ are constants independent of the data $[27,12]$. In most cases, the norms $\|\cdot\|_{1,2,3}$ are the same, while $\|\cdot\|_{4}$ is different. If the estimate (2.2) holds for $F=g=0$, the problem is called well-posed. The estimate (2.2) implies that the solution $u$ is unique, and that it depends continuously on the data $F, g$ and 
$f$. To clarify this statement, assume that $v$ is a solution to (2.1) with perturbed data $F+\delta F, g+\delta g$ and $f+\delta f$, such that,

$$
\begin{aligned}
v_{t} & =H v+F+\delta F, \quad \bar{x} \in \Omega, t \geq 0 \\
L v & =g+\delta g, \quad \bar{x} \in \delta \Omega, t \geq 0 \\
v & =f+\delta f, \quad \bar{x} \in \Omega, t=0 .
\end{aligned}
$$

Subtracting (2.1) from (2.3) results in the difference problem,

$$
\begin{aligned}
e_{t} & =H e+\delta F, \quad \bar{x} \in \Omega, t \geq 0 \\
L e & =\delta g, \quad \bar{x} \in \delta \Omega, t \geq 0 \\
e & =\delta f, \quad \bar{x} \in \Omega, t=0,
\end{aligned}
$$

where $e=v-u$. Equation (2.4) is analogous to (2.1), and therefore satisfy an estimate of the same form as (2.2), i.e.

$$
\|e\|_{1}^{2} \leq C e^{\alpha t}\left(\|\delta f\|_{2}^{2}+\int_{0}^{t}\left(\|\delta F\|_{3}^{2}+\|\delta g\|_{4}^{2}\right) d \tau\right) .
$$

We first note that if the perturbations are small, $e$ must be also be small, which means that the solution depends continuously on applied data. In particular, if $\delta f=\delta F=\delta g=0$, then $e=0$, which leads to uniqueness.

Remark 2.1.1. Note that (2.2) does not imply a bounded solution for large $t$ if $\alpha>0$, i.e. the solution is allowed to grow.

\subsubsection{A model problem}

Next, we illustrate the previous discussion by considering a model problem,

$$
u_{t}+a u_{x}=F, \quad x \in[0,1],
$$

where $a>0$ is a constant and $F$ an arbitrary forcing function. By applying the energy method to (2.5), i.e. multiplying with $u$ and integrating over the spatial domain, we get,

$$
\|u\|_{t}^{2}=a\left(u^{2}(0, t)-u^{2}(1, t)\right)+2 \int_{0}^{1} u F d x,
$$

where the norm is defined as $\|u\|^{2}=\int_{0}^{1} u^{2} d x$.

In order to obtain an estimate of the form $(2.2)$, the term $a\left(u^{2}(0)-u^{2}(1)\right)$ must be limited by applying the boundary condition $u(0, t)=g(t)$ at $x=0$. We get,

$$
\|u\|_{t}^{2} \leq a g^{2}(t)+\eta\|u\|^{2}+\frac{1}{\eta}\|F\|^{2},
$$


where the estimate $2 \int_{0}^{1} u F d x \leq \eta\|u\|^{2}+\|F\|^{2} / \eta$ for an arbitrary constant $\eta>0$ has been used. Solving (2.7) for $\|u\|^{2}$ yields,

$$
\|u\|^{2} \leq e^{\eta t}\left(\|f\|^{2}+\int_{0}^{t}\left(a g^{2}+\frac{1}{\eta}\|F\|^{2} d \tau\right)\right.
$$

and an estimate of the form (2.2) has been obtained.

Remark 2.1.2. Note that the presence of $F$ leads to the growth factor $\eta$. In this thesis, $F$ is often not present or omitted since it does not influence wellposedness.

\subsection{The discrete problem}

When using the energy method, the boundary terms are obtained through integration by parts, as illustrated in (2.6). The SBP operators mimic this procedure for the discrete problem. Hence, a SBP operator $D=P^{-1} Q$ that approximate the spatial derivative have the property,

$$
(\bar{u}, D \bar{u})_{P}+(D \bar{u}, \bar{u})_{P}=u_{n}^{2}-u_{0}^{2},
$$

where the subscript denote grid point index and $\bar{u}=\left[u_{0}, u_{1}, \ldots, u_{n}\right]^{T}$ is a grid function with the values of $u$ injected at the grid points. The inner product is defined as $(v, w)_{P}=v^{T} P w$ for two vectors $v, w$.

The property (2.9) is achieved by demanding that the matrix $Q$ satisfy the so-called SBP property,

$$
Q+Q^{T}=\operatorname{diag}(-1,0, \ldots, 0,1) .
$$

Moreover, the $r$ order accurate SBP operator $D$ must approximate derivatives of polynomials up to degree $r$ exactly, such that,

$$
D \bar{x}^{k}=k \bar{x}^{k-1}, \quad k=0,1, \ldots r
$$

where $\bar{x}^{k}=\left[x_{0}^{k}, x_{1}^{k}, \ldots, x_{n}^{k}\right]^{T}$ is a grid function of the polynomial $x^{k}$. The matrix $P$ must satisfy

$$
P=P^{T}>0,
$$

such that the discrete norm $\|\bar{u}\|_{P}^{2}=\bar{u}^{T} P \bar{u}$ approximates the continous norm $\|u\|^{2}=\int_{0}^{1} u^{2} d x$.

Similar to the condition for well-posedness, a strongly stable numerical scheme has a solution $\bar{u}$ that satisfies,

$$
\|\bar{u}\|_{1}^{2} \leq \bar{C} e^{\bar{\alpha} t}\left(\|\bar{f}\|_{2}^{2}+\int_{0}^{t}\left(\|\bar{F}\|_{3}^{2}+\|\bar{g}\|_{4}^{2}\right) d \tau\right),
$$


where $\bar{C}$ and $\bar{\alpha}$ are constants independent of the data and the mesh parameters $[27,12]$. As for the continous problem, the terms in (2.13) can be expressed in different norms. When using SBP operators, the norms $\|\cdot\|_{1,2,3}$ are in most cases equal to $\|\cdot\|_{P}$, while $\|\cdot\|_{4}$ is different. In (2.13), $\bar{F}, \bar{f}, \bar{g}$ are grid functions of $F, f$ and $g$, respectively. If the estimate (2.13) holds for $\bar{F}=\bar{g}=0$, the problem is said to be stable.

\subsubsection{The one-dimensional SBP-SAT technique}

Next, we use the SBP operators described above to approximate the spatial derivative in the model problem (2.5). To implement the boundary conditions, we use SAT's $[4,5]$. The SBP-SAT approximation of $(2.5)$ becomes,

$$
\bar{v}_{t}+a P^{-1} Q \bar{v}=\sigma P^{-1} E_{0}\left(\bar{v}_{0}-\bar{g}\right)+\bar{F} .
$$

The term $\sigma P^{-1} E_{0}\left(\bar{v}_{0}-\bar{g}\right)$ on the right-hand side of (2.14) is the SAT penalty term that enforces the boundary condition $u(0, t)=g(t)$, and the parameter $\sigma$ is the penalty parameter that has to be chosen such that the scheme is stable. In $(2.14), \bar{v}_{0}=\left[v_{0}, 0, \ldots, 0\right]^{T}$, in which $v_{0}$ denotes the numerical solution at the first grid point, and $\bar{g}=[g, 0, \ldots, 0]^{T}$.

Applying the discrete energy method to (2.14) (multiplying with $\bar{v}^{T} P$ from the left and adding the transpose of the outcome) gives,

$$
\left(\|\bar{v}\|_{P}^{2}\right)_{t}=(a+\sigma) v_{0}^{2}+\sigma\left(v_{0}-g\right)^{2}-\sigma g^{2}-a v_{n}^{2}+2 \bar{v}^{T} P \bar{F} .
$$

The right-hand side is bounded by data for $\sigma \leq-a$. In particular, $\sigma=-a$ yields,

$$
\left(\|\bar{v}\|_{P}^{2}\right)_{t}=a g^{2}-a v_{n}^{2}+2 \bar{v}^{T} P \bar{F}-a\left(v_{0}-g\right)^{2},
$$

which is completely analogous to the continous estimate (2.6) if $v_{0}=g$.

By using that $2 \bar{v}^{T} P \bar{F} \leq \eta\|\bar{v}\|_{P}^{2}+\|\bar{F}\|_{P}^{2} / \eta$ for any $\eta>0$ and solving (2.16) for $\|\bar{v}\|_{P}^{2}$ in a similar manner as in Section 2.1.1 yields the final result,

$$
\|\bar{v}\|_{P}^{2} \leq e^{\eta t}\left(\|\bar{f}\|_{P}^{2}+\int_{0}^{t}\left(a g^{2}+\frac{1}{\eta}\|\bar{F}\|_{P}^{2} d \tau\right)\right.
$$

where the initial condition $\bar{v}(0)=\bar{f}$ has been imposed. Hence, the numerical scheme (2.14) is stable. Note the similarity of the discrete estimate (2.17) to the continous one in (2.8). 


\subsubsection{The multi-dimensional SBP-SAT technique}

Consider the advection equation in two space dimensions,

$$
\begin{aligned}
u_{t}+a u_{x}+b u_{y} & =0, \quad(x, y) \in[0,1], t \geq 0 \\
u(0, y, t) & =g_{x}(y, t), \quad x=0, t \geq 0 \\
u(x, 0, t) & =g_{y}(x, t), \quad y=0, t \geq 0 \\
u(x, y, 0) & =f(x, y), \quad(x, y) \in[0,1], t=0,
\end{aligned}
$$

where $a, b>0$.

The numerical scheme that approximates (2.18) is,

$$
\begin{gathered}
\bar{v}_{t}+a\left(D_{x} \otimes I_{y}\right) \bar{v}+b\left(I_{x} \otimes D_{y}\right) \bar{v}= \\
\sigma_{x}\left(P_{x}^{-1} E_{0 x} \otimes I_{y}\right)\left(\bar{v}_{x=0}-\bar{g}_{x}\right)+ \\
\sigma_{y}\left(I_{x} \otimes P_{y}^{-1} E_{0 y}\right)\left(\bar{v}_{y=0}-\bar{g}_{y}\right) \\
\bar{v}(0)=\bar{f}
\end{gathered}
$$

where $\sigma_{x, y}$ are the penalty matrices. The solution $\bar{v}$ is arranged as $\bar{v}=\left[\bar{v}_{0}, \ldots, \bar{v}_{n_{x}}\right]$, in which $\bar{v}_{k}$ is a $n_{y} \times 1$ vector that approximates the solution along the the line $x=x_{k}$. The number of grid points in the $x$ and $y$ direction are $n_{x}$ and $n_{y}$, respectively. In (2.19), $I_{x, y}$ are identity matrices of appropriate sizes and the subscript on $D$ and $P$ denotes the derivative that is being approximated. The symbol $\otimes$ denotes the Kronecker product, which is defined as,

$$
A \otimes B=\left[\begin{array}{cccc}
A_{11} B & A_{12} B & \ldots & A_{1 m} B \\
A_{21} B & A_{22} B & \ldots & \vdots \\
\vdots & \vdots & \ddots & \vdots \\
A_{n 1} B & \ldots & \ldots & A_{n n} B
\end{array}\right]
$$

for two matrices $A, B$.

In the discrete energy method for two-dimensional problems, one multiply (2.19) with $\bar{v}^{T}\left(P_{x} \otimes P_{y}\right)$ from the left and add the transpose of the outcome. That yields,

$$
\begin{array}{r}
\left(\|\bar{v}\|_{P_{x} \otimes P_{y}}^{2}\right)_{t}=a \bar{g}_{x}^{T}\left(E_{0 x} \otimes P_{y}\right) \bar{g}_{x}-a \bar{v}_{x=1}^{T}\left(E_{N x} \otimes P_{y}\right) \bar{v}_{x=1}+ \\
b \bar{g}_{y}^{T}\left(P_{x} \otimes E_{0 y}\right) \bar{g}_{y}-b \bar{v}_{y=1}^{T}\left(P_{x} \otimes E_{N y}\right) \bar{v}_{y=1}- \\
a\left(\bar{v}_{x=0}-\bar{g}_{x}\right)^{T}\left(E_{0 x} \otimes P_{y}\right)\left(\bar{v}_{x=0}-\bar{g}_{x}\right)- \\
b\left(\bar{v}_{y=0}-\bar{g}_{y}\right)^{T}\left(P_{x} \otimes E_{0 y}\right)\left(\bar{v}_{y=0}-\bar{g}_{y}\right)
\end{array}
$$

where we have used $\sigma_{x}=-a$ and $\sigma_{y}=-b$. Equation (2.20) imply that the growth of $\bar{v}$ is limited by data and the problem is therefore stable. 



\section{The multiple penalty technique}

As pointed out previously, additional information available inside the computational domain can be used to alter and improve the numerical scheme. The MPT technique is a generalization of the SAT technique described above, and utilizes a weak implementation of the additional data. In this chapter, we show how the MPT can be used for different applications.

First, we consider the MPT applied to the model problem (2.5). Assume that the solution is known at $x=0$ (which is necessary for a well-posed problem) and at a few additional grid points inside the computational domain. As an illustration, we assume that data is available at two additional grid points close to the left boundary, see Figure 3.1. The additional data can be implemented using SAT's, such that (2.14) with $\bar{F}=0$ becomes,

$$
\begin{aligned}
\bar{v}_{t}+a P^{-1} Q \bar{v}= & \sigma_{00} P^{-1} E_{0}\left(\bar{v}_{0}-\bar{g}_{0}\right)+\sigma_{01} P^{-1} E_{0}\left(\bar{v}_{1}-\bar{g}_{1}\right)+ \\
& \sigma_{10} P^{-1} E_{1}\left(\bar{v}_{0}-\bar{g}_{0}\right)+\sigma_{11} P^{-1} E_{1}\left(\bar{v}_{1}-\bar{g}_{1}\right)+ \\
& \sigma_{12} P^{-1} E_{1}\left(\bar{v}_{2}-\bar{g}_{2}\right)+\sigma_{20} P^{-1} E_{2}\left(\bar{v}_{0}-\bar{g}_{0}\right)+ \\
& \sigma_{02} P^{-1} E_{0}\left(\bar{v}_{2}-\bar{g}_{2}\right)+\sigma_{21} P^{-1} E_{2}\left(\bar{v}_{1}-\bar{g}_{1}\right)+ \\
& \sigma_{22} P^{-1} E_{2}\left(\bar{v}_{2}-\bar{g}_{2}\right),
\end{aligned}
$$

where $\bar{v}_{j}=\left[0, \ldots, v_{j}, \ldots 0\right]^{T}, \bar{g}_{j}=\left[0, \ldots, g_{j}, \ldots 0\right]^{T}$ and $v_{j}, g_{j}$ denotes the solution and known data at grid point $j$, respectively. The elements of the matrices $E_{j}$ are zero except at element $(j, j)$, where it is one, and $\sigma_{i j}$ are penalty coefficients to be determined. By applying the discrete energy method to (3.1) with zero data, we get,

$$
\left(\|\bar{v}\|_{P}^{2}\right)_{t}=\bar{v}_{B}^{T}\left(\Sigma+\Sigma^{T}\right) \bar{v}_{B}-a v_{N}^{2},
$$

where $\bar{v}_{B}=\left[v_{0}, v_{1}, v_{2}\right]$ and,

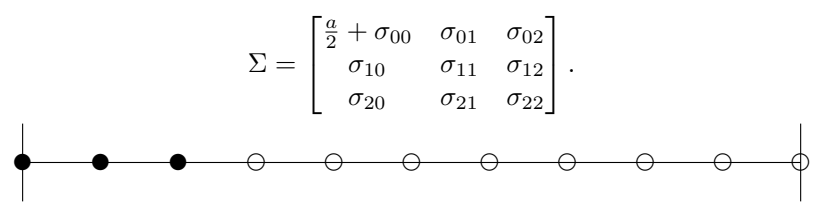

Figure 3.1: Illustration of the mesh in one space dimension. The circles denotes the grid points and filled circles denotes grid points where SAT's are applied. 
For stability, the penalty coefficients must be chosen such that the right-hand side (3.1) is non-positive, i.e. such that $\Sigma+\Sigma^{T} \leq 0$. As long as that condition is fulfilled, the coefficents $\sigma_{i j}$ are arbitrary. In the upcoming sections, we shall show how to improve the scheme in different ways by choosing these coefficents wisely.

\subsection{Increasing the rate of convergence}

Adding additional penalties like the ones in (3.1) adds damping to the scheme, which can be used to increase the rate of convergence. This section is based on Paper I, but we will do a more careful analysis than provided in that paper. To illustrate, we consider the exact solution $\bar{u}$ injected into (3.1) with all offdiagonal penalty coefficents equal to zero,

$$
\begin{array}{r}
\bar{u}_{t}+a P^{-1} Q \bar{u}=\sigma_{00} P^{-1} E_{0}\left(\bar{u}_{0}-\bar{g}_{0}\right)+\sigma_{11} P^{-1} E_{1}\left(\bar{u}_{1}-\bar{g}_{1}\right)+ \\
\sigma_{22} P^{-1} E_{2}\left(\bar{u}_{2}-\bar{g}_{2}\right)+T e,
\end{array}
$$

where $T e$ is the truncation error. Subtracting (3.1) from (3.3) yields the error equation,

$$
\begin{array}{r}
\bar{e}_{t}+a P^{-1} Q \bar{e}=\sigma_{00} P^{-1} E_{0} \bar{e}_{0}+\sigma_{11} P^{-1} E_{1} \bar{e}_{1}+ \\
\sigma_{22} P^{-1} E_{2} \bar{e}_{2}+T e,
\end{array}
$$

where $\bar{e}=\bar{u}-\bar{v}$. Applying the discrete energy method to (3.4) results in,

$$
\left(\|\bar{e}\|_{P}^{2}\right)_{t}=\left(a+2 \sigma_{00}\right) e_{0}^{2}+2 \sigma_{11} e_{1}^{2}+2 \sigma_{22} e_{2}^{2}+2 \bar{e}^{T} P T e .
$$

Using that $\left(\|\bar{e}\|_{P}^{2}\right)_{t}=2\|\bar{e}\|_{P}\left(\|\bar{e}\|_{P}\right)_{t}$ and $2 \bar{e}^{T} P T e \leq 2\|\bar{e}\|_{P}\|T e\|_{P}$ leads to,

$$
\left(\|\bar{e}\|_{P}\right)_{t} \leq-\eta(t)\|\bar{e}\|_{P}+\|T e\|_{P}
$$

where

$$
\eta(t)=-\frac{\left(a+2 \sigma_{00}\right) e_{0}^{2}+2 \sigma_{11} e_{1}^{2}+2 \sigma_{22} e_{2}^{2}}{2\|\bar{e}\|^{2}} .
$$

If the MPT is applied at many grid points, if $\sigma_{00} \leq-a / 2$ and all additional penalty coefficients are negative (which is necessary for stability), the function $\eta(t)$ will be large, resulting in a rapid decay of the error, as seen in (3.6).

To clarify, assume that the error is large at $t=0$, when the simulation is initiated. Solving (3.6) for $\|\bar{e}\|_{P}$ yields,

$$
\|\bar{e}\|_{P} \leq e^{-K(t)}\|\bar{e}(0)\|_{P}+e^{-K(t)} \int_{0}^{t} e^{K(\tau)}\|T e\|_{P} d \tau,
$$

where $K(t)=\int_{0}^{t} \eta(\tau) d \tau$ is the primitive function of $\eta(t)$ and $\bar{e}(0)$ is the initial error. The function $K(t)$ will be large when the MPT is applied at many grid points (since $\eta(t)$ is large), and the initial error, $\bar{e}(0)$, will decay rapidly. 


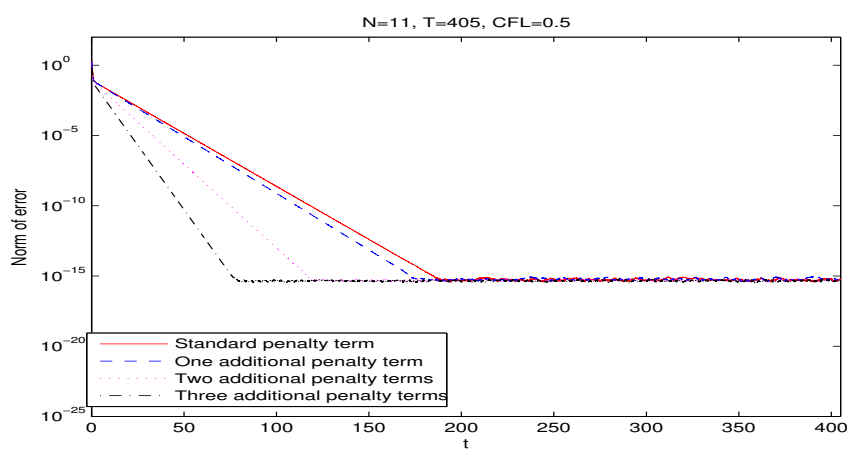

Figure 3.2: The P-norm of the error when using standard SAT's and up to three additional penalty terms.

In Figure 3.2, the model problem (2.5) with $F=0$ and $a=1$ is solved when using additional penalty parameters close to the left boundary. The total simulation time is $T=405$, the initial condition is $u(x, 0)=1+e^{-100(x-0.5)^{2}}$, the grid spacing is $\Delta x=1 / 10$ and the CFL number is $C F L=\Delta t / \Delta x=0.5$. The error should converge to zero as $t \rightarrow \infty$. One can clearly see that the rate of convergence is increased when the MPT is applied.

\subsection{Increasing the accuracy}

In order for the SBP finite difference operator to satisfy property (2.10), the order of accuracy close to the boundaries is lower than in the interior of the domain. In this thesis, we consider diagonal $P$ 's, and the corresponding finite difference operator is $2 p$ order accurate in the interior and $p$ order accurate close to the boundaries [16]. In the next application of the MPT introduced in Paper I, we will choose the penalty coefficients in (3.1) such as the finite difference operator has the same order everywhere while the SBP property (2.10) is preserved.

Consider the finite difference operator $P^{-1} \tilde{Q}$, where $\tilde{Q}$ is a uniformly $2 p$ order accurate operator. The SBP property $(2.10)$ is no longer satisfied, and a rest term $\delta Q$ will be present on the right hand side,

$$
\tilde{Q}+\tilde{Q}^{T}=B+\delta Q
$$

where $B=\operatorname{diag}(-1,0, \ldots, 0,1)$. The matrix $\delta Q$ is zero except at elements close to the boundaries. 
Consider (3.1) with zero data and with the operator $P^{-1} \tilde{Q}$ used for approximating the spatial derivative,

$$
\bar{v}_{t}+a P^{-1} \tilde{Q} \bar{v}=\sigma_{00} P^{-1} E_{0} \bar{v}_{0}+P^{-1} \Sigma \bar{v},
$$

where all penalty terms except the first one are collected in the term $P^{-1} \Sigma \bar{v}$. The following analysis is also valid for non-zero data, but including such data will obscure the main points of this section. The discrete energy method on (3.8) yields,

$$
\left(\|\bar{v}\|_{P}^{2}\right)_{t}=-a v_{N}^{2}+\left(a+2 \sigma_{00}\right) v_{0}^{2}+\bar{v}^{T}\left(-\delta Q+\Sigma^{T}+\Sigma\right) \bar{v},
$$

and we observe that the scheme is stable if,

$$
\begin{aligned}
\sigma_{00} & \leq-\frac{a}{2} \\
-\delta Q+\Sigma^{T}+\Sigma & =0 .
\end{aligned}
$$

In particular, if $\sigma_{00}=-a / 2,(3.9)$ mimics the continous estimate (2.6) with $u(0, t)=F=0$. In summary: by choosing the penalty coefficients according to (3.10), a uniformly $2 p$ order accurate finite difference operator can be used while the scheme remains stable. One can also add on damping by requiring that the last relation in (3.10) is negative semi-definite.

Next, we illustrate the results above by consider a uniformly second order accurate operator $D=P^{-1} \tilde{Q}$, where,

$$
\tilde{Q}=\left[\begin{array}{cccccc}
-\frac{3}{4} & 1 & -\frac{1}{4} & \cdots & & 0 \\
-\frac{1}{2} & 0 & \frac{1}{2} & & & \vdots \\
\vdots & & & \ddots & & \\
& & & -\frac{1}{2} & 0 & \frac{1}{2} \\
0 & \ldots & & \frac{1}{4} & -1 & \frac{3}{4}
\end{array}\right],
$$

and $P=\Delta x \operatorname{diag}(1 / 2,1, \ldots, 1,1 / 2)$. The matrix $\tilde{Q}$ does not satisfy the SBP property (2.10), and we get,

$$
\tilde{Q}+\tilde{Q}^{T}=\left[\begin{array}{cccccc}
-\frac{3}{2} & \frac{1}{2} & -\frac{1}{4} & \ldots & & 0 \\
\frac{1}{2} & 0 & 0 & & & \vdots \\
-\frac{1}{4} & 0 & 0 & & & \\
\vdots & & & \ddots & & \\
& & & 0 & 0 & \frac{1}{4} \\
0 & \ldots & & \frac{1}{4} & -\frac{1}{2} & \frac{3}{2}
\end{array}\right], \delta Q=\left[\begin{array}{cccccc}
\frac{1}{2} & \frac{1}{2} & -\frac{1}{4} & \ldots & & 0 \\
\frac{1}{2} & 0 & 0 & & \\
-\frac{1}{4} & 0 & 0 & & \\
\vdots & & & \ddots & \\
& & 0 & 0 & \frac{1}{4} \\
& & & 0 & 0 & -\frac{1}{2} \\
0 & \ldots & & \frac{1}{4} & -\frac{1}{2} & -\frac{1}{2}
\end{array}\right] .
$$

Hence, in order to make the last term on the right hand side of (3.9) zero, we 
choose,

$$
\Sigma=\frac{1}{2}\left[\begin{array}{cccccc}
\frac{1}{2} & \frac{1}{2} & -\frac{1}{4} & \ldots & & 0 \\
\frac{1}{2} & 0 & 0 & & & \vdots \\
-\frac{1}{4} & 0 & 0 & & & \\
\vdots & & & \ddots & & \\
& & & 0 & 0 & \frac{1}{4} \\
& & & 0 & 0 & -\frac{1}{2} \\
0 & \ldots & & \frac{1}{4} & -\frac{1}{2} & -\frac{1}{2}
\end{array}\right] .
$$

\subsection{Modifying the wave speed of the error}

Consider the error equation related to (3.1),

$$
\bar{e}_{t}+a P^{-1} Q \bar{e}=\sigma_{00} P^{-1} E_{0} \bar{e}_{0}+P^{-1} \Sigma \bar{e} .
$$

As in the previous section, all additional penalty terms have been collected in the term $P^{-1} \Sigma \bar{e}$. Note that (3.11) describes the error in a traveling wave with wave speed $a$.

To proceed, we rearrange (3.11),

$$
\bar{e}_{t}+P^{-1}(a Q-\Sigma) \bar{e}=\sigma_{00} P^{-1} E_{0} \bar{e}_{0},
$$

and let the structure of the matrix $a Q-\Sigma$ be,

$$
a Q-\Sigma=\left[\begin{array}{ccc}
a Q_{L}-\Sigma_{L} & 0 & 0 \\
0 & Q_{I} & 0 \\
0 & 0 & a Q_{R}-\Sigma_{R}
\end{array}\right]
$$

By choosing $\Sigma_{L}=\alpha_{L} Q_{L}$ and $\Sigma_{R}=\alpha_{R} Q_{R}$, the wave speed at the boundary regions will then be modified to $\left(a-\alpha_{L, R}\right)$, respectively. A more detailed discussion on how to alter the wave speed of the error can be found in Paper I.

\subsection{Constructing absorbing boundary layers}

As described in Section 3.1, the MPT has a damping effect, that reduces the errors. By applying the MPT when an outgoing wave is about to hit the boundary, it can be damped out and thus preventing unwanted reflections. In Paper I, the following procedure is applied to hyperbolic systems. Here, we will describe the procedure by considering a scalar equation, for simplicity.

Consider the model problem (2.5) with zero boundary data, zero forcing function and the initial condition $u(x, 0)=e^{-200(x-0.5)^{2}}$ in the domain $x \in[0,1]$. The 

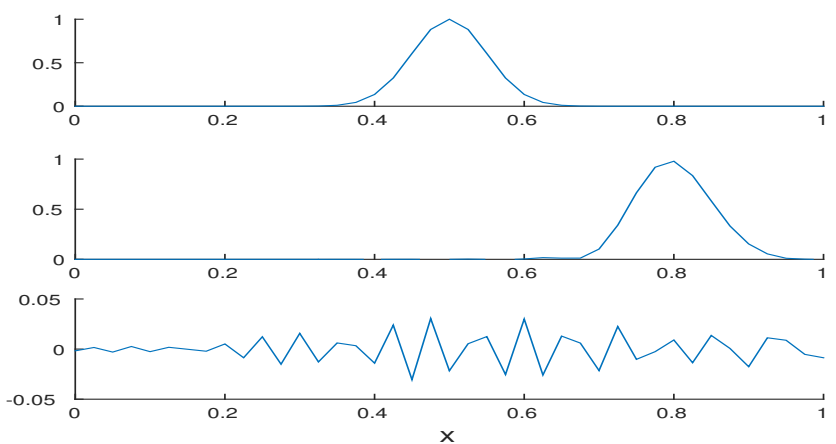

Figure 3.3: The solution to (2.5) at $t=0$ (upper), $t=0.3$ (middle) and $t=0.9$ (lower) when using standard penalty terms.

problem is integrated up to $t=0.9$ using standard penalties, $N=40$ grid points in space and a third order SBP-SAT scheme for the spatial discretization. Snap shots of the solution are displayed in Figure 3.3. Note that the outgoing wave is partly reflected at the boundary $x=1$.

Next, we apply the MPT at all grid points in the region $[0.7,1]$ when $t=0.3$ (when the wave is inside the penalty region) to damp out the outgoing wave. The results are displayed in Figure 3.4, and one can see that the reflections are significantly reduced.

\subsection{The MPT in several space dimensions}

In the examples above, the MPT has been applied to problems in a single space dimension. In this section, we apply the MPT on multidimensional problems, which is based on the results of Paper II. For our purposes, it suffice to consider a hyperbolic system in two space dimensions,

$$
\begin{aligned}
u_{t}+A u_{x}+B u_{y} & =0, \quad(x, y) \in[0,1] \\
L_{x} u(0, y, t) & =g_{x 0}(y, t) \\
R_{x} u(1, y, t) & =g_{x 1}(y, t) \\
L_{y} u(x, 0, t) & =g_{y 0}(x, t) \\
R_{y} u(x, 1, t) & =g_{y 1}(x, t) \\
u(x, y, 0) & =f(x, y)
\end{aligned}
$$



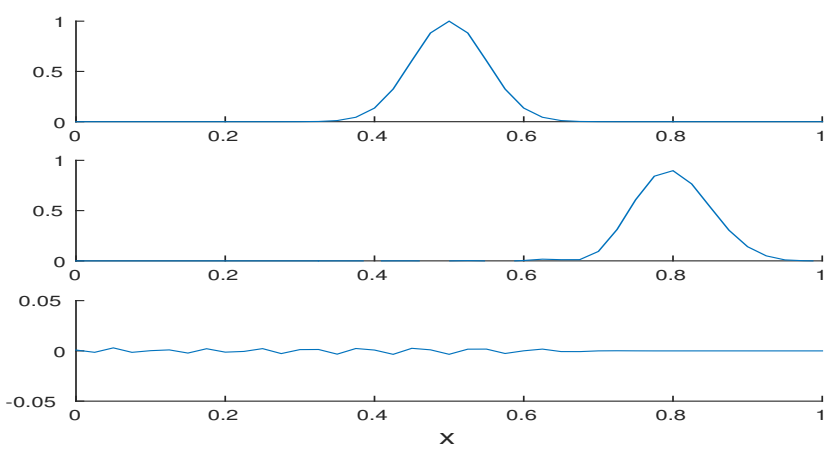

Figure 3.4: The solution to (2.5) at $t=0$ (upper), $t=0.3$ (middle) and $t=0.9$ (lower) when using the MPT.

where $A, B$ are constant and symmetric matrices, $L_{x, y}$ and $R_{x, y}$ are appropriate boundary operators that result in a well-posed problem and $g_{x 0}, g_{x 1}, g_{y 0}, g_{y 1}$ and $f(x, y)$ are given boundary and initial data.

Consider the case where additional data is known at a few additional points inside the spatial domain, and denote the set of these points $\Omega_{s}$. In $\Omega_{s}$, we assume that there are operators $L_{i j}$ and known data $g_{i j}$ that satisfies the relation

$$
L_{i j} u\left(x_{i}, y_{j}, t\right)=g_{i j}(t), \quad\left(x_{i}, y_{j}\right) \in \Omega_{s} .
$$

By using the SBP-SAT technique described in Section 2.2.2 to discretize (3.12) and utilizing the MPT to implement the additional data results in,

$$
\begin{array}{r}
\bar{v}_{t}+\left(D_{x} \otimes I_{y} \otimes A\right) \bar{v}+\left(I_{x} \otimes D_{y} \otimes B\right) \bar{v}= \\
\left(P_{x}^{-1} E_{0 x} \otimes I_{y} \otimes \Sigma_{L x} L_{x}\right) \bar{v}+\left(P_{x}^{-1} E_{N x} \otimes I_{y} \otimes \Sigma_{R x} R_{x}\right) \bar{v}+ \\
\left(I_{x} \otimes P_{y}^{-1} E_{0 y} \otimes \Sigma_{L y} L_{y}\right) \bar{v}+\left(I_{x} \otimes P_{y}^{-1} E_{N y} \otimes \Sigma_{R y} R_{y}\right) \bar{v}+ \\
\sum_{x_{i}, y_{j} \in \Omega_{s}}\left(P_{x}^{-1} E_{i x} \otimes P_{y}^{-1} E_{j y} \otimes \Sigma_{i j} L_{i j}\right) \bar{v}
\end{array}
$$

where $\bar{v}$ is the numerical solution. For simplicity, all the data, including the additional part, are set to zero. The finite difference operators $D_{x, y}$ are on SBP form, $I_{x, y}$ identity matrices of appropriate sizes and $\Sigma_{L, R x}, \Sigma_{L, R y}, \Sigma_{i j}$ are penalty matrices to be determined such that (3.13) is stable. The elements of the matrices $E_{i x, y}$ are zero, except at element $(i, i)$, where they are equal to one.

Remark 3.5.1. The following derivation is also valid for non-zero data, but including such data will obscure the main points of this section. 
Applying the discrete energy method to (3.13) (multiplying with $\bar{v}^{T}\left(P_{x} \otimes P_{y} \otimes I\right)$ and adding the transpose of the outcome), results in,

$$
\begin{array}{r}
\left(\|\bar{v}\|_{P x \otimes P_{y} \otimes I}^{2}\right)_{t}=\bar{v}^{T}\left(E_{0 x} \otimes P_{y} \otimes\left(A+\Sigma_{L x} L_{x}+\left(\Sigma_{L x} L_{x}\right)^{T}\right)\right) \bar{v}+ \\
\bar{v}^{T}\left(E_{N x} \otimes P_{y} \otimes\left(-A+\Sigma_{R x} R_{x}+\left(\Sigma_{R x} R_{x}\right)^{T}\right)\right) \bar{v}+ \\
\bar{v}^{T}\left(P_{x} \otimes E_{0 y} \otimes\left(B+\Sigma_{L y} L_{y}+\left(\Sigma_{L y} L_{y}\right)^{T}\right)\right) \bar{v}+ \\
\bar{v}^{T}\left(P_{x} \otimes E_{N y} \otimes\left(-B+\Sigma_{R y} R_{y}+\left(\Sigma_{R y} R_{y}\right)^{T}\right)\right) \bar{v}+ \\
\sum_{x_{i}, y_{j} \in \Omega_{s}} \bar{v}^{T}\left(E_{i x} \otimes E_{j y} \otimes\left(\Sigma_{i j} L_{i j}+\left(\Sigma_{i j} L_{i j}\right)^{T}\right)\right) \bar{v}
\end{array}
$$

For stability, the right-hand side of (3.14) must be non-positive. Consequently, the penalty matrices at the boundaries must be chosen such that,

$$
\begin{aligned}
& A+\Sigma_{L x} L_{x}+\left(\Sigma_{L x} L_{x}\right)^{T} \leq 0, \quad-A+\Sigma_{R x} R_{x}+\left(\Sigma_{R x} R_{x}\right)^{T} \leq 0 \\
& B+\Sigma_{L y} L_{y}+\left(\Sigma_{L y} L_{y}\right)^{T} \leq 0, \quad-B+\Sigma_{R y} R_{y}+\left(\Sigma_{R y} R_{y}\right)^{T} \leq 0,
\end{aligned}
$$

and the penalty matrices associated with the MPT must satisfy,

$$
\Sigma_{i j} L_{i j}+\left(\Sigma_{i j} L_{i j}\right)^{T} \leq 0 .
$$

Note that one can always find non-zero $\Sigma_{i j}$ such that (3.15) is fulfilled. For example, the choice $\Sigma_{i j}=\beta L_{i j}^{T}$ satisfies (3.15) for any $\beta<0$.

\subsection{Controlling error growth}

Many problems experience an error growth in time, even though the IBVP is well-posed and the scheme is stable. For example, consider the advection equation with periodic boundary conditions,

$$
\begin{aligned}
u_{t}+u_{x} & =0, \quad x \in[0,1] \\
u(0, t) & =u(1, t) \\
u(x, 0) & =f(x) .
\end{aligned}
$$

The corresponding numerical scheme using the SBP-SAT technique is,

$$
\bar{v}_{t}+D \bar{v}=-\frac{1}{2} P^{-1} E_{0}\left(\bar{v}_{0}-\hat{v}_{N}\right)+\frac{1}{2} P^{-1} E_{N}\left(\bar{v}_{N}-\hat{v}_{0}\right),
$$

where $\hat{v}_{N}=\left[v_{N}, 0, \ldots, 0\right]^{T}$ and $\hat{v}_{0}=\left[0, \ldots, 0, v_{0}\right]^{T}$. Inserting the exact solution into (3.17) and subtracting it from (3.17) with the numerical solution $\bar{v}$ results in the error equation,

$$
\bar{e}_{t}+D \bar{e}=-\frac{1}{2} P^{-1} E_{0}\left(\bar{e}_{0}-\hat{e}_{N}\right)+\frac{1}{2} P^{-1} E_{N}\left(\bar{e}_{N}-\hat{e}_{0}\right)+T e,
$$


where $T e$ is the truncation error. Applying the discrete energy method and following a similar procedure as in Section 3.1 results in,

$$
\left(\|\bar{e}\|_{P}\right)_{t} \leq\|T e\|_{P}
$$

By integrating in time, one can conclude that,

$$
\|\bar{e}\|_{P} \leq\|\bar{e}(0)\|_{P}+t\|T e\|_{P}^{\max }
$$

where $\|T e\|_{P}^{\max }$ is an upper estimate of $\|T e\|_{P}$. As one can see, the periodic boundary conditions result in a linear growth of the error. Consequently, the error is unbounded for long time simulations.

Now, consider the following situation. First we solve (3.17) up to $t=t_{0}$, next we apply the MPT, such that (3.18) becomes,

$$
\bar{e}_{t}+D \bar{e}=-\frac{1}{2} P^{-1} E_{0}\left(\bar{e}_{0}-\hat{e}_{N}\right)+\frac{1}{2} P^{-1} E_{N}\left(\bar{e}_{N}-\hat{e}_{0}\right)+\sum_{x_{i} \in \Omega_{s}} \sigma_{i i} P^{-1} E_{i} \bar{e}+T e .
$$

By applying the discrete energy method we find,

$$
\left(\|\bar{e}\|_{P}\right)_{t} \leq-\eta(t)\|\bar{e}\|_{P}+\|T e\|_{P}^{\max },
$$

where $\eta(t)=-\sum_{x_{i} \in \Omega_{s}} \sigma_{i i} e_{i}^{2} /\left(\|\bar{e}\|_{P}^{2}\right)$. If the penalty coefficents are chosen appropriately, we may assume that $\eta(t) \geq \eta_{0}>0$, and we obtain

$$
\|\bar{e}\|_{P} \leq e^{-\eta_{0}\left(t-t_{0}\right)}\left\|\bar{e}\left(t_{0}\right)\right\|_{P}+\frac{1-e^{-\eta_{0}\left(t-t_{0}\right)}}{\eta_{0}}\|T e\|_{P}^{\max }, \quad t \geq t_{0} .
$$

First, we note that an error bound is obtained by applying the MPT. Secondly, the initial error, $\left\|\bar{e}\left(t_{0}\right)\right\|_{P}$, decays exponentially. In summary, the error grows linearly in the time interval $t \in\left[0, t_{0}\right]$, where only standard SAT's are used. When the MPT is applied at $t=t_{0}$, the accumulated error will decay rapidly, and hence, the error growth can be controlled by using the MPT.

We illustrate the theoretical results above by considering an example from Paper III. The problem (3.16) is solved using the grid parameters $\Delta x=1 / 40$ and $\Delta t=1 / 100$. Additional penalty terms are applied at $N_{M P T}=10$ spatial grid points in the time intervals $t \in[5,7], t \in[15,17]$ and $t \in[25,27]$. The total simulation time is $T=30$. In Figure 3.5, both the function $\eta$, defined in (3.21), and the P-norm of the error is displayed as a function of time. When the MPT is applied, $\eta$ becomes quite large, resulting in a rapid decay of the error. Consequently, the error can be kept below a desired level by occasionally applying the MPT. In Paper III, the analysis described above is applied to the linearized shallow water equations, yielding similar results.

\subsection{Conclusions and future outlook}

The MPT is a simple and flexible technique that has many areas of application, and can be applied to most problems. If the original scheme is stable and 

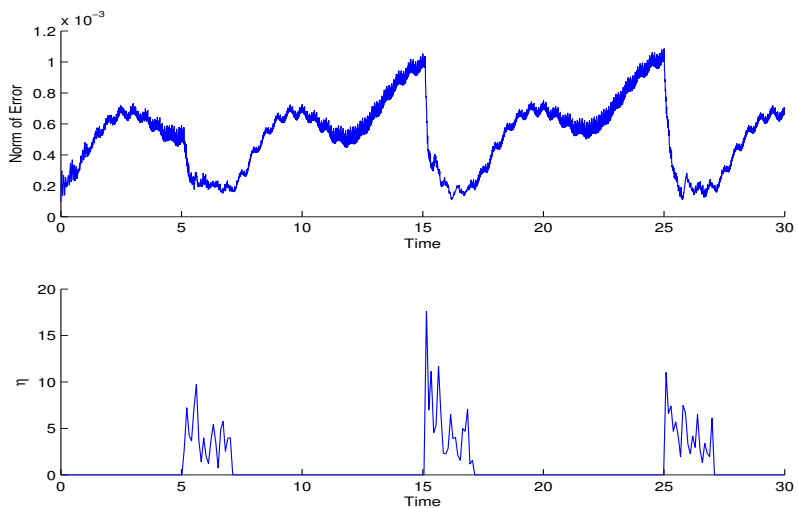

Figure 3.5: The function $\eta$ and the P-norm of the error as a function of time.

accurate, the MPT can be applied while the scheme remains stable and accurate. Similar techniques for incorporating additional data are, for example, Davies relaxation [7] and Newtonian nudging [2]. Compared to these techniques, the main advantage of the MPT is its simplicity, and that it always result in a provable stable scheme.

At the current state, all data is assumed to be exact when applying the MPT; that is, it does not take into account that the data may be inaccurate. This is often the case in realistic computations. Therefore, an interesting subject for future studies would be to include uncertainty in the applied data when using the MPT. 


\section{Non-reflecting boundary conditions}

NRBC's are used for limiting otherwise infinite physical domains. As we will see in this chapter, constructing NRBC's for one-dimensional hyperbolic problems is rather straight forward. In two dimensions, however, the problem becomes significantly more complicated. To begin with, we provide an overview of the theory behind NRBC's and clarify by considering problems in one space dimension. We proceed by describing the technique introduced in Paper IV, where NRBC's for multi-dimensional problems are considered.

\subsection{NRBC's in one dimension}

Consider the wave equation in one space dimension,

$$
u_{t t}=c^{2} u_{x x}, \quad x \in[0,1], \quad t \geq 0,
$$

where $c$ is a positive constant. For our purposes, it suffice to consider solutions to (4.1) in the form of traveling waves, such that (4.1) have solutions on the form,

$$
u=\sigma_{+} e^{i \omega(c t+x)}+\sigma_{-} e^{i \omega(c t-x)},
$$

where $\sigma_{ \pm}$are determined by the boundary conditions. The term $u_{+}=e^{i \omega(c t+x)}$ represent a mode traveling to the left, i.e. towards the boundary $x=0$, while the mode $u_{-}=e^{i \omega(c t-x)}$ travels to the right, towards the boundary $x=1$. For either boundary to be non-reflecting, only modes traveling towards the boundaries can be allowed. In our case, the boundary $x=0$ must only allow for the mode $u_{+}$and the boundary $x=1$ must only allow for the mode $u_{-}$.

Assume that we want the boundary $x=0$ to be non-reflecting; that is, the boundary condition must only allow for incoming modes, i.e. the mode $u_{+}$. This is realized by constructing a boundary condition that enforces $\sigma_{-}=0$. The NRBC at $x=0$ can then be written,

$$
u_{t}(0, t)-c u_{x}(0, t)=0 .
$$


Since $u_{t}-c u_{x}=2 i \omega c \sigma_{-} e^{i \omega c t}$, the boundary condition (4.3) demands that $\sigma_{-}=$ 0 . Similarly, the NRBC at $x=1$ is,

$$
u_{t}(1, t)+c u_{x}(1, t)=0 .
$$

The NRBC's for (4.1) can also be obtained by applying the Laplace transform, such that (4.1) with zero initial data becomes,

$$
s^{2} \hat{u}=c^{2} \hat{u}_{x x},
$$

where $s$ is the dual variable to $t$. Inserting the ansatz $\hat{u}=e^{k x}$ into (4.5) yields the solution,

$$
\hat{u}(x)=\sigma_{+} e^{\frac{s}{c} x}+\sigma_{-} e^{-\frac{s}{c} x} .
$$

The NRBC's are obtained by removing all modes that decays for $\operatorname{Re}(s)>0$ at $x=0$ and all modes that grows at $x=1$, such that $\sigma_{-}=0$ at $x=0$ and $\sigma_{+}=0$ at $x=1$. The above conditions are achieved by the boundary conditions,

$$
\begin{array}{ll}
s \hat{u}-c \hat{u}_{x}=0, & x=0 \\
s \hat{u}+c \hat{u}_{x}=0, & x=1,
\end{array}
$$

which results in the boundary conditions (4.3) and (4.4) after transforming back to the time-domain.

To illustrate the results above, consider a similar setup as in Section 3.4. First, the problem (4.1) with $c=1$ is solved using the reflecting boundary conditions,

$$
u_{x}(0, t)=0, \quad u_{x}(1, t)=0,
$$

and the initial condition $u(x, 0)=e^{-200(x-0.5)^{2}}$. Snap shots of the solution is shown in Figure 4.1. At the lower figure, the waves have been reflected at both boundaries. Next, the simulation described above is repeated using the NRBC's (4.3) and (4.4), and the results are displayed in Figure 4.2. The reflections observed in Figure 4.1 are no longer present.

\subsection{NRBC's for systems of hyperbolic equations}

Next, we discuss how to construct NRBC's for hyperbolic systems of equations in two space dimensions, i.e. problems on the form,

$$
u_{t}+A u_{x}+B u_{y}=0, \quad(x, y) \in[0,2] \times[0,1], \quad t \geq 0
$$

where $A, B$ are symmetric matrices. We restrict the analysis to the boundaries $y=\{0,1\}$, and therefore assume that the spatial domain is periodic in the $x$-direction. A detailed description of how to construct continous NRBC's for (4.6) can be found in $[9,25]$, and we will do a similar analysis here. 

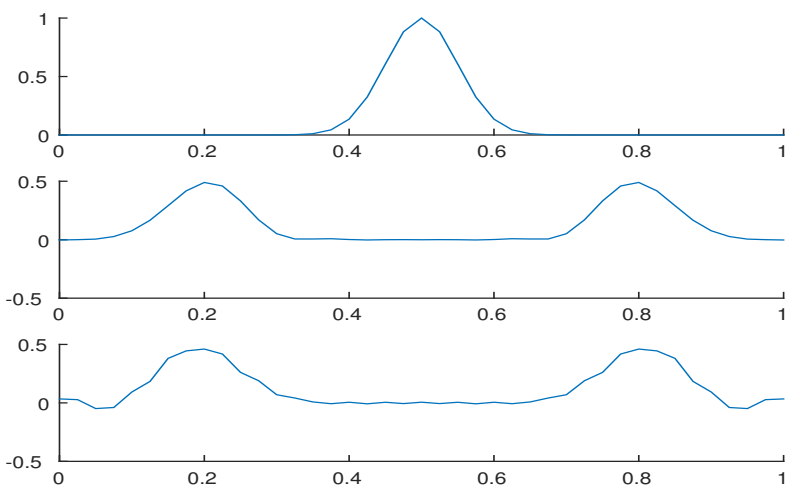

Figure 4.1: The solution to (4.1) at $t=0$ (upper), $t=0.3$ (middle) and $t=0.8$ (lower) using reflecting boundary conditions.
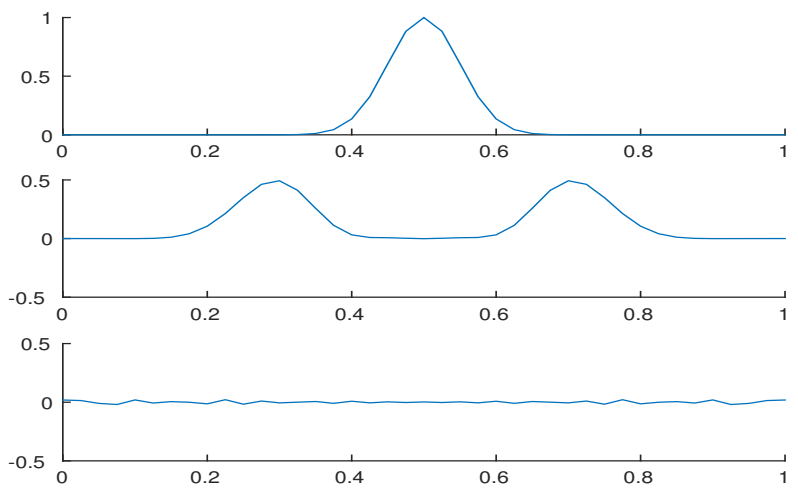

Figure 4.2: The solution to (4.1) at $t=0$ (upper), $t=0.3$ (middle) and $t=0.8$ (lower) using NRBC's. 
The first step in the construction of NRBC's is to apply the Laplace transform in time and Fourier transform in the $x$-direction, such that (4.6) becomes

$$
s \hat{u}+i \omega A \hat{u}+B \hat{u}_{y}=0,
$$

where $s$ and $\omega$ are the dual variables to $t$ and $x$, respectively. We assume that the initial data is zero. Assuming that $B$ is non-singular and rearranging (4.6) gives us,

$$
\hat{u}_{y}+M(s, \omega) \hat{u}=0,
$$

where $M(s, \omega)=s B^{-1}+i \omega B^{-1} A$.

Inserting the ansatz $\hat{u}=e^{-k y} \psi(s, \omega)$ into (4.8) results in the eigenvalue problem,

$$
(M(s, \omega)-k I) \psi(s, \omega)=0 .
$$

Obviously, $k$ coincides with the eigenvalues of $M$ and $\psi(s, \omega)$ are the corresponding eigenvectors. The solution to (4.7) can now be written as a linear combination of eigenvectors to $M$,

$$
\hat{u}=\sum_{j} \sigma_{j} e^{-k_{j}(s, \omega) y} \psi_{j}(s, \omega)=\Psi e^{-K y} \bar{\sigma},
$$

where the $\sigma_{j}$ 's are determined by the boundary conditions. In (4.10), $\Psi=$ $\left[\psi_{1}, \ldots, \psi_{n}\right]$ is the matrix of eigenvectors, $K=\operatorname{diag}\left(k_{1}, \ldots, k_{n}\right)$ and $\bar{\sigma}=\left[\sigma_{1}, \ldots, \sigma_{n}\right]^{T}$ is the vector of coefficients.

To annihilate all downwards traveling modes at $y=0$ (i.e. all modes with positive real part of $k$ ), we must construct boundary conditions that forces the corresponding coefficients $\sigma_{j}$ to zero, i.e.

$$
\sigma_{j}(s, \omega)=0, \quad \operatorname{Re}\left(k_{j}\right)>0, y=0 .
$$

In a similar way, the NRBC's at $y=1$ must result in,

$$
\sigma_{j}(s, \omega)=0, \quad \operatorname{Re}\left(k_{j}\right)<0, y=1 .
$$

The requirements $(4.11),(4.12)$ can be realized by the boundary conditions,

$$
M^{+} \hat{u}(0)=0, \quad M^{-} \hat{u}(1)=0 .
$$

In (4.13), $M^{ \pm}=\Psi K^{ \pm} \Psi^{-1}$ and $K^{ \pm}$is the part of $K$ with positive and negative real part, respectively. Inserting (4.10) into (4.13) leads to the conditions (4.11) and (4.12).

Arriving at the NRBC's (4.13) is relatively easy. However, when implementing them, they need to be transformed back to the physical space. The required back transformation is the major obstacle, that prevent the use of this technique for most practical flow problems. 


\subsubsection{NRBC's for one-dimensional hyperbolic problems}

We illustrate the results above by considering the simplified case where $A=0$, such that (4.6) becomes a one-dimensional problem,

$$
u_{t}+B u_{y}=0
$$

The matrix $M(s, \omega)=M(s)$ can then be simplified as,

$$
M(s)=s B^{-1},
$$

and we conclude that the eigenfunctions $\psi$ are constant and equal to the eigenvectors of $B$. The general solution to (4.14) in Laplace space is,

$$
\hat{u}=\sum_{j} \sigma_{j} e^{-\frac{s}{\lambda_{j}} y} \psi_{j},
$$

where $\lambda_{j}$ are the eigenvalues $B, \psi_{j}$ the corresponding eigenvectors and $\sigma_{j}$ coefficients determined by the boundary conditions.

In order to make the boundary $y=0$ non-reflecting, all decaying modes must vanish, i.e.

$$
\sigma_{j}(s, \omega)=0, \quad \lambda_{j}>0, y=0,
$$

and the corresponding condition at the boundary is,

$$
\sigma_{j}(s, \omega)=0, \quad \lambda_{j}<0, y=1 .
$$

The above conditions can be enforced by the boundary conditions,

$$
B^{+} \hat{u}(0)=0, \quad B^{-} \hat{u}(1)=0,
$$

where $B^{ \pm}$denotes the positive and negative part of $B$, respectively.

Since $B^{ \pm}$are independent of $s$, the relation (4.15) can easily be transformed back into real space, to yield

$$
B^{+} u(0)=0, \quad B^{-} u(1)=0 .
$$

The relation (4.16) is recognized as the characteristic boundary conditions to (4.14).

\subsection{The new way to construct NRBC's}

As mentioned above, implementing the continous NRBC's is a major issue as they are expressed in a transformed space. In Paper IV, we introduce a technique that circumvent this issue by using SBP operators for the time discretization, and we briefly describe that technique in this section. 
First, consider (4.6) with the initial condition $u(x, y, 0)=0$, discretized in time and the $x$-direction using the SBP-SAT technique,

$$
\left(\tilde{D}_{t} \otimes I_{x} \otimes B^{-1}\right) \bar{v}+\left(I_{t} \otimes D_{x} \otimes B^{-1} A\right) \bar{v}+\bar{v}_{y}=0 .
$$

In (4.17), we have multiplied the problem with $B^{-1}$ from the left. The operator $\tilde{D}_{t}=P_{t}^{-1}\left(Q_{t}+E_{0 t}\right)$ includes the SBP finite difference operator and the penalty term that implements the initial condition. The results in [23] show that the eigenvalues of $\tilde{D}_{t}$ has a strictly positive real part. With periodic boundary conditions, the spatial difference operator is skew symmetric, i.e. $D_{x}=-D_{x}^{T}$.

Next, the matrices $\tilde{D}_{t}=X_{t} \hat{S} X_{t}^{-1}$ and $D_{x}=i X_{x} \hat{\Omega} X_{x}^{*}$ are diagonalized. Note that $\operatorname{Re}(\hat{S})>0$ and that $D_{x}$ has purely imaginary eigenvalues. Also, $D_{x}$ is diagonalized by a unitary matrix $X_{x}$, since it is skew symmetric. By multiplying (4.17) with $X_{t}^{-1} \otimes X_{x}^{*} \otimes I$ from the left, we obtain,

$$
\hat{v}_{y}+\hat{M} \hat{v}=0
$$

where $\hat{v}=\left(X_{t}^{-1} \otimes X_{x}^{*} \otimes I\right) \bar{v}$ and,

$$
\hat{M}=\left[\begin{array}{cccc}
M\left(\hat{s}_{0}, \hat{\omega}_{0}\right) & 0 & \ldots & 0 \\
0 & M\left(\hat{s}_{0}, \hat{\omega}_{0}\right) & \ldots & \vdots \\
\vdots & \ldots & \ddots & \vdots \\
0 & \ldots & \ldots & M\left(\hat{s}_{N_{t}}, \hat{\omega}_{N_{x}}\right)
\end{array}\right] .
$$

The matrix blocks $M$ is the same matrix as in (4.8) and $\hat{s}_{i}, \hat{\omega}_{i}$ are the diagonal elements of $\hat{S}$ and $\hat{\Omega}$, respectively.

As in Section 4.2, the NRBC's are given by,

$$
\hat{M}^{+} \hat{v}(0)=0, \quad \hat{M}^{-} \hat{v}(1)=0 .
$$

Unlike the boundary conditions derived in Section 4.2, the boundary conditions (4.19) are given in the discrete setting, and transforming back to the original variables is straight forward. One simply multiply the problem with $X_{t} \otimes X_{x} \otimes I$ from the left.

The boundary conditions (4.19) can be implemented directly using penalty terms, such that (4.18) becomes,

$$
\hat{v}_{y}+\hat{M} \hat{v}=L_{y=0}\left(\hat{B}^{-1} \Sigma_{0} \hat{M}^{+} \hat{v}\right)+L_{y=1}\left(\hat{B}^{-1} \Sigma_{1} \hat{M}^{-} \hat{v}\right),
$$

in which $\hat{B}=\left(I_{t} \otimes I_{x} \otimes B\right)$ and $L_{y=y_{0}}$ are so-called lifting operators, that for two arbitrary functions $\alpha, \beta$ satisfies,

$$
\int_{0}^{1} L_{y=y_{0}}(\alpha \beta)=\alpha\left(y_{0}\right) \beta\left(y_{0}\right) .
$$




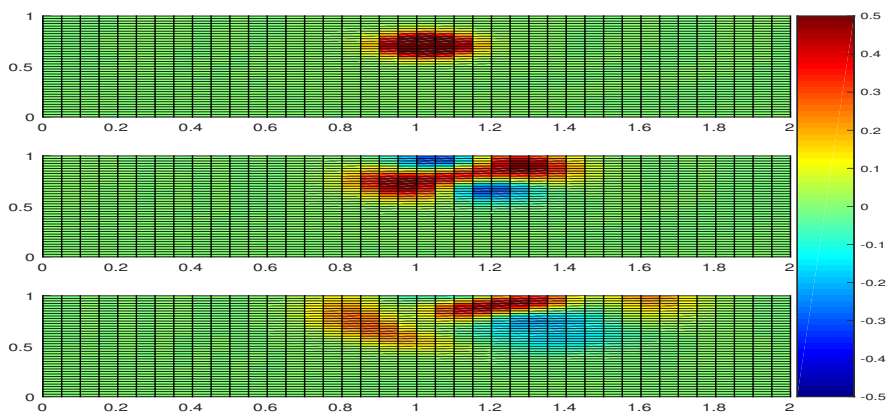

Figure 4.3: The reference solution $u_{1}$ at different time levels using the grid $\Delta x=2 / 40$, $\Delta y=1 / 40$. Upper: $t=0$, middle: $t=0.2$ and bottom: $t=0.4$. A third order SBP scheme has been used.

In Paper IV, we show that the matrices $\Sigma_{0,1}$ can be chosen in several ways to produce a stable scheme, and the least complicated form is

$$
\Sigma_{0}=-\hat{B} \hat{M}^{-1}, \quad \Sigma_{1}=\hat{B} \hat{M}^{-1} .
$$

Once the penalty matrices are obtained, the problem can easily be transformed back into the original variables and solved.

\subsubsection{Numerical verification}

To verify the theoretical results discussed above, a reference solution is created by solving (4.6) on a large domain and for a sufficiently small simulation time, such that the reflections from the boundaries are not present in the regular domain at the final time. The matrices $A, B$ used in the computation are,

$$
A=\left[\begin{array}{ccc}
u & c / \sqrt{2} & -c / \sqrt{2} \\
c / \sqrt{2} & u & 0 \\
-c / \sqrt{2} & 0 & u
\end{array}\right], \quad B=\left[\begin{array}{ccc}
v & 0 & 0 \\
0 & v-c & 0 \\
0 & 0 & v+c
\end{array}\right],
$$

where $u, v$ is the horizontal and vertical component of a reference state velocity, respectively, and $c$ is the gravity wave speed. Equation (4.6) with $A, B$ given above are the linearized shallow water equations. The first component, $u_{1}$, of the reference solution at different time levels is shown in Figure 4.3.

Secondly, (4.6) is solved with the NRBC's (4.19), implemented as in (4.20) using the penalty matrices (4.21). The error is the defined as the deviation from the 


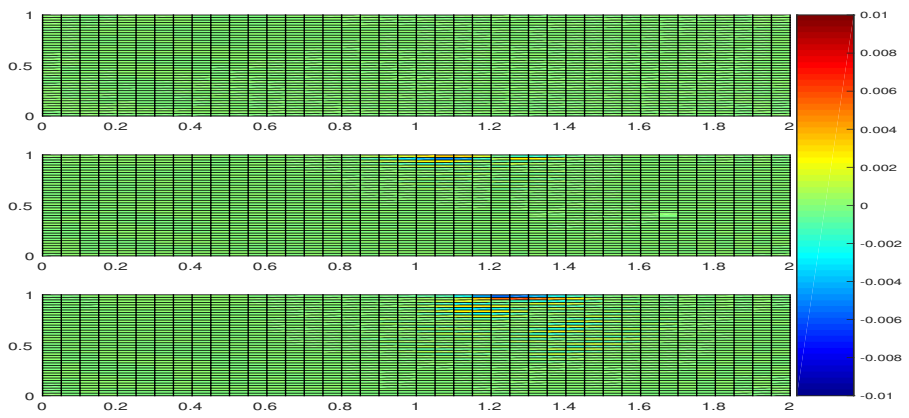

Figure 4.4: The error of the component $u_{1}$ at different time levels using the grid $\Delta x=$ $2 / 40, \Delta y=1 / 40$ and the NRBC's (4.19). Upper: $t=0$, middle: $t=0.2$ and bottom: $t=0.4$. A third order SBP scheme has been used.

\begin{tabular}{c|c|c||c|c||c|c}
$\mathrm{N}$ & $\mathrm{SBP}(2,1)$ & Rate & $\mathrm{SBP}(4,2)$ & Rate & $\mathrm{SBP}(6,3)$ & Rate \\
\hline 12 & $2.79 \cdot 10^{-2}$ & - & $5.57 \cdot 10^{-2}$ & - & $1.41 \cdot 10^{-1}$ & - \\
20 & $1.55 \cdot 10^{-2}$ & 1.2 & $1.95 \cdot 10^{-2}$ & 2.1 & $4.02 \cdot 10^{-2}$ & 2.5 \\
30 & $8.00 \cdot 10^{-3}$ & 1.6 & $6.64 \cdot 10^{-3}$ & 2.7 & $1.13 \cdot 10^{-2}$ & 3.1 \\
40 & $4.56 \cdot 10^{-3}$ & 2.0 & $3.13 \cdot 10^{-3}$ & 2.6 & $3.56 \cdot 10^{-3}$ & 4.0 \\
50 & $2.92 \cdot 10^{-3}$ & 2.0 & $1.39 \cdot 10^{-3}$ & 3.6 & $1.28 \cdot 10^{-3}$ & 4.6
\end{tabular}

Table 4.1: The P-norm of the error at $t=0.4$ for different mesh-sizes when using a second $(\operatorname{SBP}(2,1))$, third $(\operatorname{SBP}(4,2))$ and fourth $(\operatorname{SBP}(6,3))$ order SBP scheme.

reference solution. In Figure 4.4, the error is displayed, and one can observe that almost no reflections appear close to the boundary $y=1$.

In Table 4.1 , the $\mathrm{P}$-norm of the error at $t=0.4$ is shown when using a second $(\mathrm{SBP}(2,1))$, third $(\mathrm{SBP}(4,2))$ and fourth $(\mathrm{SBP}(6,3))$ order accurate $\mathrm{SBP}$ scheme for the temporal and spatial discretization. As one can see, the rate of convergence during mesh refinement is as expected, which means that the boundary conditions (4.19) only produces numerical errors.

\subsection{Conclusions and future outlook}

In Paper IV, we have introduced a provable stable and accurate way to impose exact non-reflecting boundary conditions. The theory behind the procedure is relatively straight forward, and the implementation is not an issue. The 
numerical experiments show that the reflections are of the same size as the truncation error, which verifies that the NRBC's are indeed non-reflecting.

The boundary conditions (4.19) are global, i.e. they include the solution from all previous time steps. Consequently, solving the problem in practice is computationally demanding, and calculations on fine grids during long time intervals is currently an issue. Future problems to be solved therefore include the constructing of an efficient parallel algorithm for solving (4.20). Moreover, one could also consider making suitable approximations that would reduce the computational effort, as well as investigating a multi-block version in time. 



\section{Error bounds for the wave equation}

As briefly discussed in Section 3.6, the error for most hyperbolic problems grows during long time simulations. In paper $\mathrm{V}$, error bounds for the wave equation on second order form is investigated, and we will shortly discuss that paper in this chapter.

We consider the wave equation in one space dimension, including non-reflecting boundary conditions and suitable initial conditions,

$$
\begin{aligned}
u_{t t} & =c^{2} u_{x x} \\
u_{t}(0, t)-u_{x}(0, t) & =g_{0}(t) \\
u_{t}(1, t)+u_{x}(1,1) & =g_{1}(t) \\
u_{t}(x, 0) & =f(x) \\
u(x, 0) & =h(x) .
\end{aligned}
$$

In (5.1), $c>0$ is a constant and $g_{0}, g_{1}, f, h$ are given boundary and initial data. By using the SBP-SAT technique, the semi-discrete version of (5.1) becomes,

$$
\begin{aligned}
\bar{v}_{t t} & =c^{2} D \bar{v}_{x}-c P^{-1} E_{0}\left(\bar{v}_{t}-c \bar{v}_{x}-\bar{g}_{0}\right)-c P^{-1} E_{N}\left(\bar{v}_{t}+c \bar{v}_{x}-\bar{g}_{1}\right) \\
\bar{v}(0) & =\bar{f} \\
\bar{v}_{t}(0) & =\bar{h}
\end{aligned}
$$

in which $\bar{g}_{0}, \bar{g}_{1}, \bar{f}, \bar{h}$ are grid functions of $g_{0}, g_{1}, f, h$, respectively.

The semi-discrete error equation is obtained by inserting the exact solution into (5.2) and subtracting (5.2) from it. We find,

$$
\begin{aligned}
\bar{e}_{t t} & =c^{2} D \bar{e}_{x}-c P^{-1} E_{0}\left(\bar{e}_{t}-c \bar{e}_{x}\right)-c P^{-1} E_{N}\left(\bar{e}_{t}+c \bar{e}_{x}\right)+T e \\
\bar{e}(0) & =0 \\
\bar{e}_{t}(0) & =0
\end{aligned}
$$

where $T e$ is the truncation error and $\bar{e}=\bar{u}-\bar{v}$. As described in Paper $\mathrm{V}$ (see also $[29,18])$, the energy method applied to (5.3) leads to the estimate,

$$
\frac{\partial}{\partial t}\left(\left\|\bar{e}_{t}\right\|_{P}^{2}+c^{2}\left\|\bar{e}_{x}\right\|_{P}^{2}\right) \leq-c\left(e_{t N}^{2}+e_{t 0}^{2}\right)+2\left\|\bar{e}_{t}\right\|\|T e\|_{P}
$$


Contrary to the cases studied in previous chapters, (5.4) yields a bound on $\bar{e}_{t}$ and $\bar{e}_{x}$, rather than the actual error $\bar{e}$. Consequently, one can prove that the error growth is limited, but not that it is bounded.

To clarify, we let $\|\tilde{e}\|_{P}^{2}=\left\|\bar{e}_{t}\right\|_{P}^{2}+c^{2}\left\|\bar{e}_{x}\right\|_{P}^{2}$ and observe that (5.4) can be rewritten as,

$$
\|\tilde{e}\|_{P} \leq-\eta(t)\|\tilde{e}\|_{P}+\|T e\|_{P}^{\max },
$$

where,

$$
\eta(t)=c \frac{e_{t N}^{2}+e_{t 0}^{2}}{2\|\tilde{e}\|_{P}^{2}} \geq \eta_{0}>0 .
$$

In (5.5) $\|T e\|_{P}^{\max }$ is an upper estimate of $\|T e\|_{P}$. By solving (5.5) for $\|\tilde{e}\|_{P}$, we find

$$
\|\tilde{e}\|_{P} \leq \frac{1-\exp \left(-\eta_{0} t\right)}{\eta_{0}}\|T e\|_{P}^{\max },
$$

where the relation $\eta(t) \geq \eta_{0}>0$ has been used. By (5.6), we can conclude that,

$$
\left\|\bar{e}_{t}\right\|_{P} \leq \frac{1-\exp \left(-\eta_{0} t\right)}{\eta_{0}}\|T e\|_{P}^{\max }, \quad\left\|\bar{e}_{x}\right\|_{P} \leq \frac{1-\exp \left(-\eta_{0} t\right)}{\eta_{0}}\|T e\|_{P}^{\max } .
$$

However, no bound for the error $\bar{e}$ is obtained.

The theoretical findings above indicate that the error grows linearly in time, see Paper V for details. However, as we will see, numerical experiments indicate that the error is in fact bounded. In Paper V, we perform numerical tests for several cases, and the error turns out to be bounded during long time simulations for all of them. As an illustration, we include one of these tests. The total simulation time is set to $T=100$, the exact solution is $u=\sin (2 \pi(x-t))$, and the data is chosen accordingly. A SBP scheme of third order overall accuracy is used for both the temporal and spatial discretization. The space and time derivative of the error together with the error itself of (5.2) is displayed in Figure 5.1. The time and space derivatives of the error are bounded, as predicted above. In contrast with the theoretical results, the error turns out to be bounded as well, indicating that the derived theoretical estimates are non-sharp.

\subsection{Conclusions and future outlook}

Error bounds for the wave equation has been derived in similar way as in $[22,17]$. In contrast to the hyperbolic problems on first order form previously studied, no bound of the actual error can be obtained. One can prove that the derivatives of the error are bounded, which suggest that the actual error grows at a linear rate. As the growth of the error is contradicted by numerical experiments, the error bounds derived are probably not optimal. In future studies, a more accurate error estimate should be derived. 

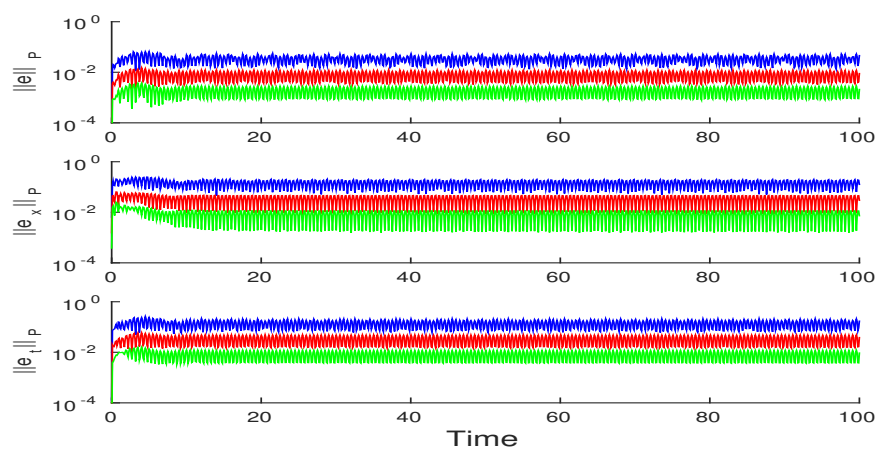

Figure 5.1: The P-norm of $\bar{e}$ (upper), $\bar{e}_{x}$ (middle) and $\bar{e}_{t}$ (lower) as a function of time when solving (5.2) using a third order SBP scheme. 



\section{Summary of papers}

\section{Paper I: A flexible boundary procedure for hyper- bolic problems: multiple penalty terms applied in a domain}

The MPT technique is introduced and applied to one-dimensional hyperbolic problems. First and foremost, we investigate how the additional data should be implemented without ruining the stability of the scheme. Once stability is ensured, we show how to use the free parameters to

- increase the rate of convergence.

- create boundary zones with non-reflecting properties.

- raise the accuracy.

- modify the wave speed of the error.

The technique is applied to both the advection equation and systems of hyperbolic equations, such as the linearized Euler and elastic wave equation. The theoretical findings are confirmed with numerical experiments.

\section{Paper II: A stable and accurate Davies-like relax- ation procedure using multiple penalty terms for lateral boundary conditions}

In the weather prediction community, inter-spaced local domains are often used to capture different phenomena with high accuracy. For this reason, a finer grid than in the global domain is required. In this paper, we introduce a procedure for incorporating the global data into the local domains in a stable and accurate way using the SAT technique. The procedure generalizes the MPT discussed in Paper I to several space dimensions. 
The technique is tested on the shallow water equations for several choices of boundary data, and the results show that the MPT reduces the errors in the local domain and increases the rate of convergence, both for steady state calculations and time dependent problems.

\section{Paper III: A stable and accurate data assimilation technique using multiple penalty terms in space and time}

In Paper I and II, the additional data is assumed to be known during the entire simulation time when applying the MPT. However, in many scenarios, the data is only known during limited time intervals. In this paper, we further extend the technique proposed in Paper I and II, by showing how MPT's can be applied during limited time intervals without ruining accuracy and stability.

The method is demonstrated on the advection equation in one space dimension and the linearized shallow water equations. In both cases, periodic boundary conditions are applied, which results in a linear growth of the error without the MPT. It is shown that the error growth is prevented by applying the MPT, and one can keep the error below a desired level by occasionally implementing additional data during limited time intervals.

\section{Paper IV: Constructing non-reflecting boundary conditions using summation-by-parts in time}

NRBC's for hyperbolic systems in two space dimensions has been investigated. First, we show that the NRBC's result in a well-posed problem. Next, by using SBP operators for the time integration, we introduce a way to circumvent the implementation issues normally experienced when considering NRBC's. The NRBC's are derived directly for the discrete problem, and the implementation is therefore not a problem. We also show that the derived boundary conditions result in a stable and accurate scheme, and that they are numerical versions of the NRBC's in the continous setting. As the entire analysis is performed in the discrete time-domain, the NRBC's are derived without applying the Laplace transform to the problem. Hence, the issues of transforming back to computational space can be avoided by using our technique.

The technique is applied to the linearized shallow water equations, and it is found that the reflections are of the same order of magnitude as the truncation error, which show that the derived NRBC's only produces numerical errors. 


\section{Paper V: Long time error for the wave equation on second order form}

Error bounds for the wave equation for long time simulations is investigated. The energy method applied the wave equation does not lead to a bound on the solution, but rather a bound on its spatial and temporal derivative. These result implies that the error can grow at most linearly in time. However, numerical experiments indicate that an error bound exist. 


\section{References}

[1] S. Abarbanel, D. Gottlieb, and J.S. Hesthaven. Well-posed perfectly matched layers for advective acoustics. Journal of Computational Physics, 154:266-283, 1999.

[2] R. Anthes. Data assimilation and initialization of hurricane prediction models. Journal of the Atmospheric Sciencies, 31:702-719, 1973.

[3] J.P. Berenger. A perfectly matched layer for the absorption of electromagnetic waves. Journal of Computational Physics, 114:185-200, 1994.

[4] M. Carpenter, D. Gottlieb, and S. Abarbanel. Time-stable boundary conditions for finite-difference schemes solving hyperbolic systems: methodology and application to high-order compact schemes. Journal of Computational Physics, 111:220-236, 1994.

[5] M. Carpenter, J. Nordström, and D. Gottlieb. A stable and conservative interface treatment of arbitrary spatial accuracy. Journal of Computational Physics, 148:341-365, 1999.

[6] P. Courtier, E. Andersson, W. Heckley, D. Vasiljevic, M. Hamrud, A. Hollingsworth, F. Rabier, M. Fisher, and J. Pailleux. The ECMWF implementation of three-dimensional variational assimilation (3D-Var) I: formulation. Quarterly Journal of the Royal Meteorological Society, 124:17831807, 1998.

[7] H. C. Davies. A lateral boundary formulation for multi-level prediction models. Quarterly Journal of the Royal Meteorological Society, 102:406418, 1976.

[8] J. Derber and F. Bouttier. A reformulation of the background error covariance in the ECMWF global data assimilation system. Tellus, 51:195-221, 1999.

[9] B. Engquist and A. Majda. Absorbing boundary conditions for the numerical simulation of waves. Mathematics of computation, 31:629-651, 1977.

[10] S. Eriksson and J. Nordström. Exact non-reflecting boundary conditions revisted: well-posedness and stability. Foundations of Computational Mathematics, February 2016. doi: 10.1007/s10208-016-9310-3.

[11] D. Givoli. High-order local non-reflecting boundary conditions: a review. Wave Motion, 39:319-326, 2004.

[12] B. Gustafsson. High order difference methods for time dependent PDE. Springer Verlag, 2008. 
[13] B. Gustafsson, H.O. Kreiss, and A. Sundström. Stability theory of difference approximations for mixed initial boundary value problems II. Mathematics of Computation, 26:649-686, 1972.

[14] T. Hagstrom. Radiation boundary conditions for the numerical simulation of waves. Acta Numerica, 8:47-106, 1999.

[15] J.S. Hesthaven. On the analysis and construction of perfectly matched layers for the linearized Euler equations. Journal of Computational Physics, 142:129-147, 1998.

[16] J.E. Hicken and D.W. Zingg. Summation-by-parts operators and highorder quadrature. Journal of Computational and Applied Mathematics, 237:111-125, 2013.

[17] D. Kopriva, J. Nordström, and G. Gassner. Error boundedness of discontinuous Galerkin spectral element approximations of hyperbolic problems. Accepted in Journal of Scientific Computing, 2016.

[18] H.O. Kriess, N.A. Petersson, and J. Yström. Difference approximations for the second order wave equation. SIAM Journal of Numerical Analysis, 40:1940-1967, 2002 .

[19] H.O. Kriess and G. Scherer. Finite element and finite difference methods for hyperbolic partial differential equations. Mathematical Aspects of Finite Elements in Partial Differential Equations, 33:195-212, 1974.

[20] T. Lundquist and J. Nordström. The SBP-SAT technique for initial value problems. Journal of Computational Physics, 270:86-104, 2014.

[21] K. Mattsson and J. Nordström. Summation by parts for finite difference approximations of second derivatives. Journal of Computational Physics, 199:503-540, 2004.

[22] J. Nordström. Error bounded schemes for time-dependent hyperbolic problems. SIAM Journal of Scientific Computing, 30:46-59, 2007.

[23] J. Nordström and T. Lundquist. Summation-by-parts in time. Journal of Computational Physics, 251:487-499, 2013.

[24] J. Nordström and T. Lundquist. Summation-by-parts in time: the second derivative. SIAM Journal of Scientific Computing, 38:A1561-A1586, 2016.

[25] C.W. Rowley and T. Colonius. Discretely non-reflecting boundary conditions for linear hyperbolic systems. Journal of Computational Physics, 157:500-538, 2000.

[26] B. Strand. Summation by parts for finite difference approximations for $\frac{d}{d x}$. Journal of Computational Physics, 110:47-67, 1994. 
[27] M. Svärd and J. Nordström. Review of summation-by-parts schemes for initial-boundary-value problems. Journal of Computational Physics, 268:17-38, 2014.

[28] S. Tsynkov. Numerical solution of problems on unbounded domains. A review. Applied Numerical Mathematics, 27:465-532, 1998.

[29] S. Wang and G. Kriess. Convergence of summation-by-parts finite difference methods for the wave equation. Accepted in Journal of Scientific Computing, 2016. 


\section{Papers}

The articles associated with this thesis have been removed for copyright reasons. For more details about these see:

http://urn.kb.se/resolve? urn:nbn:se:liu:diva-134127 